\section{Características tumorais e sobrevida de cinco anos em pacientes com câncer de mama admitidas no Instituto Nacional de Câncer, Rio de Janeiro, Brasil}

\author{
Tumor characteristics and five-year survival \\ in breast cancer patients at the National \\ Cancer Institute, Rio de Janeiro, Brazil
}

Gulnar Azevedo e Silva Mendonça ${ }^{1}$

Aline Moraes da Silva 1

Wagner Manoel Caula 1

\title{
Introdução
}

Numerous factors have been studied to establish more secure prognostic criteria in breast cancer patients. This study estimates five-year survival rates and principal prognostic factors related to tumor characteristics in women with invasive breast cancer and submitted to surgery at the National Cancer Institute, Rio de Janeiro, Brazil, from May 1995 to July 1996. Study variables were: lymph node status, tumor size, aggressiveness grade, and presence of estrogen and progesterone receptors. Survival functions were calculated according to the Kaplan-Meyer method. The Cox proportional hazards model was used to evaluate prognostic factors. Five-year survival was 75\% for all women and 64\% for those with node involvement. Multivariate analyses identified node involvement as the strongest predictor of outcome; a positive estrogen receptor test was associated with a better prognosis. These findings highlight the need for studies to assess new variables to be added to known factors in order to better orient therapy for breast cancer.

Breast Neoplasm; Prognosis; Women's Health
O câncer de mama é o tipo de neoplasia maligna mais comum entre mulheres em todo o mundo e sua ocorrência varia amplamente entre regiões. As populações que apresentam maior risco encontram-se na Europa Ocidental e nos Estados Unidos, enquanto que populações asiáticas têm taxas quase cinco vezes menores 1 . No entanto, nas últimas décadas, no Japão, vem sendo observado aumento na incidência bem como em outras regiões caracterizadas como de baixa ocorrência, como China e Índia. Ao mesmo tempo em que a incidência tende, com algumas exceções, a aumentar, a mortalidade, em países desenvolvidos (Estados Unidos, Canadá, Reino Unido, Holanda, Dinamarca e Noruega), vem declinando em cortes sucessivos de idade. No Brasil, esse tipo de câncer representa a primeira causa de óbito por câncer em mulheres, e os coeficientes de mortalidade padronizados por idade mostram tendência ascendente entre 1979 e 19992.

A queda da mortalidade verificada em países desenvolvidos foi muito rápida para ser explicada apenas pelo uso da mamografia. Chama a atenção o fato de que esse declínio não está sendo acompanhado de aumento importante nas taxas de sobrevida 3 . Isso indica que, apesar dos avanços diagnósticos e terapêuticos, ainda não se observou impacto significativo no aumento da sobrevida das pacientes. $\mathrm{Na}$ 
Europa, a sobrevida de cinco anos teve melhora em todos os países, em todos os grupos etários, menos no mais jovem (15-44 anos) 4 . Nos Estados Unidos, o decréscimo da mortalidade por câncer de mama entre mulheres brancas parece estar relacionado à difusão da mamografia e da utilização de terapias adjuvantes. Esses benefícios, todavia, não foram observados entre mulheres negras, uma vez que entre essas a incidência e a mortalidade continuam a aumentar 5. Acredita-se que o pior prognóstico entre mulheres negras possa ser explicado em parte, mas não totalmente, pelo estado avançado em que as pacientes são diagnosticadas, o que teria relação com a idade, o retardo de diagnóstico, o baixo nível sócio-econômico e a obesidade.

Vários fatores têm sido estudados no intuito de que sejam estabelecidos critérios que possam precisar, de forma mais objetiva, o prognóstico dos casos. Apesar de ser raro o aparecimento da doença antes dos 35 anos - a grande parte dos casos é diagnosticada após a menopausa - são nítidas as diferenças de prognóstico em função da idade: mulheres jovens evoluem, em geral, pior quando comparadas a mulheres mais idosas mesmo quando os dois grupos etários recebem assistência semelhante 6 .

Com respeito a características dos tumores, já é bem estabelecido que a recorrência da doença é maior em pacientes que apresentam comprometimento ganglionar axilar e que aumenta em função do número de linfonodos histologicamente positivos. Embora a dissecção dos gânglios da cadeia axilar tenha valor prognóstico, em termos terapêuticos, não acrescenta benefício, principalmente para mulheres que têm resultado negativo e acabam tendo problemas decorrentes da cirurgia alargada 7 . Novas técnicas menos invasivas, como, por exemplo, o mapeamento de linfonodos sentinelas, vêm sendo incentivadas na perspectiva de auxiliar na informação prognóstica 8,9.

Dentre os fatores prognósticos, relativos ao tumor, mais analisados destacam-se: o tamanho do tumor, o grau histológico, grau nuclear, a existência de necrose, a expressão de receptores de estrogênio e progesterona, marcadores de invasão ou capacidade de metástase, cinética de crescimento e divisão celular, e existência de vascularização 7,10. A análise combinada desses fatores pode oferecer uma alternativa menos agressiva para as mulheres do que o exame da cadeia ganglionar axilar. Infelizmente, as técnicas para acessar esses fatores inerentes ao tumor ainda não se encontram to- talmente validadas e disponíveis, e, por isso, o esvaziamento axilar continua sendo a conduta padronizada para decisão terapêutica para os casos com tumores grandes ou invasivos.

Este estudo teve como objetivo avaliar a sobrevida de cinco anos e os fatores preditores de pior prognóstico relativos ao tumor em mulheres com câncer invasivo de mama admitidas no Serviço de Mastologia do Hospital de Câncer do Instituto Nacional de Câncer (INCA), Rio de Janeiro, entre maio de 1995 e julho de 1996.

\section{Material e métodos}

\section{População de estudo}

Foi constituída pelo grupo de casos com câncer invasivo de mama que fizeram parte de estudo caso-controle 11 e que foram submetidas à cirurgia. Essas pacientes foram recrutadas no Serviço de Mastologia do Hospital de Câncer do INCA entre maio de 1995 e julho de 1996 e preenchiam os seguintes critérios de elegibilidade: serem residentes da Região Metropolitana do Rio de Janeiro por um período de no mínimo seis meses, idade até 75 anos completos, diagnóstico de câncer de mama num período inferior a seis meses quando da entrada do estudo e apresentarem condições clínicas ou emocionais para responderem os questionários.

\section{Coleta de informações}

Todas as pacientes incluídas no estudo foram entrevistadas individualmente por entrevistadores treinados que utilizaram questionário estruturado e pré-testado para levantamento de fatores de risco. Após a entrevista, as mulheres foram pesadas e tiveram sua estatura medida.

As informações relativas ao seguimento dessas pacientes foram registradas em ficha clínica, elaborada especificamente para o estudo, as quais eram preenchidas a partir de revisão de prontuários. Foram coletados os dados relativos ao estadiamento clínico, resultado do exame anatomopatológico, tipo de tratamento realizado e evolução clínica existentes no prontuário médico. Todos os laudos histopatológicos dos casos incluídos foram revistos especificamente para este estudo por duas médicas patologistas do Serviço de Anatomia Patológica do INCA.

Nos casos em que se constatou abandono de tratamento, tentou-se contato telefônico para localização da paciente e obtenção de infor- 
mação sobre o estado vital. Para os casos não localizados por telefone, foi realizada pesquisa de busca por meio do nome, data de nascimento e local de residência no Sistema de Mortalidade do Departamento de Dados Vitais da Secretaria de Estado de Saúde do Rio de Janeiro.

$\mathrm{O}$ estudo contou com 170 pacientes, sendo que dessas foi possível se constatar que 46 (27,1\% das pacientes estudadas) foram a óbito antes de completarem cinco anos de seguimento. Desses óbitos, 44 (25,9\% das pacientes estudadas) foram por neoplasia, e apenas dois casos (4,3\% dos óbitos) foram por causas não relacionadas à doença maligna; um caso foi devido a infarto agudo de miocárdio, e outro, a aneurisma dissecante de aorta. Esses dois casos, para fins de análise, não foram considerados como falhas, e a data da censura foi a do óbito. As informações sobre data e causa de óbito foram obtidas diretamente dos prontuários médicos para 31 pacientes. Apenas para um caso, esses dados foram informados por familiares através de contato telefônico. Para quatorze pacientes, as datas e causas dos óbitos foram resgatadas através da busca realizada no Sistema de Mortalidade da Secretaria Estadual de Saúde.

Após toda a fase de coleta de dados nos registros hospitalares e tentativas de busca, não foi possível se obter informação sobre seguimento de cinco anos para 28 pacientes $(16,5 \%$ do total estudado); entre essas perdas, 16 pacientes $(57,1 \%)$ foram acompanhadas por período superior a quatro anos. Para todos esses casos, a data de censura correspondeu à última data de contato constante no prontuário.

\section{Variáveis estudadas}

Foram consideradas no estudo as seguintes variáveis referentes a características dos tumores disponíveis nos registros médicos: presença de linfonodo comprometido por neoplasia, tamanho do tumor, grau de agressividade e presença de receptores hormonais para estrogênio e progesterona. O tamanho do tumor foi dicotomizado entre tumores até dois centímetros e maiores. Para caracterização de agressividade tumoral, foi utilizada a classificação de Elston 12, que considera como grau I os tumores bem diferenciados, grau II os levemente diferenciados e grau III os pouco diferenciados. Para a análise, os tumores foram divididos tomando-se por base os graus I e II comparados ao grau III. Como indicador de nível sócio-econômico, foi utilizado o nível de escolaridade no qual as mulheres foram classificadas como analfabetas ou tendo completado os seguintes níveis de ensino: elementar, fundamental, médio e superior.

\section{Análise dos dados}

Para a análise de sobrevida, foram considerados como falha os óbitos (data do óbito) em decorrência do câncer de mama ou conseqüência do tratamento. Foram censuradas, ao final do estudo, as pacientes que permaneceram vivas até 31 de julho de 2001, período que compreendia seis meses após a data de confirmação diagnóstica da última paciente incluída no estudo. Para as mulheres perdidas de seguimento, a data da censura foi a que constava no último registro do prontuário médico. As pacientes que foram a óbito por causas não relacionadas ao câncer de mama ou a seu tratamento foram censuradas na data do óbito.

Foram calculadas as funções de sobrevida empregando-se o método de Kaplan-Meier 13, no qual foram estimadas curvas agrupando as pacientes segundo as variáveis selecionadas para o estudo. O teste Log-rank foi empregado para comparação das funções de sobrevida para cada variável.

Para avaliação dos fatores prognósticos associados ao tumor, foram calculados as hazard ratios (HR) com intervalos de confiança de 95\%, seguindo-se o modelo de riscos proporcionais de Cox 14 Para a idade e nível de escolaridade, foram criadas variáveis categorizadas, e, além dos HR, foram calculados ainda os respectivos valores de $\mathrm{p}$ para o teste de $\chi^{2}$ para tendência linear. Para as variáveis relativas a características tumorais estudadas, foram calculados os HR brutos, e todas essas, por preencherem os critérios de importância clínica com base na literatura, foram selecionadas para modelo multivariado, o qual incluiu ainda a idade categorizada em grupos de cinco em cinco anos e o nível de escolaridade conforme descrito anteriormente. A proporcionalidade dos modelos de Cox foi verificada a partir do teste diagnóstico de resíduos de Schoenfeld 15 . Toda a análise foi feita utilizando-se o programa estatístico STATA, módulo ST, versão 7 .

\section{Resultados}

A média de idade das mulheres estudadas foi de 56,6 anos, e a mediana foi de 58 anos. Das 170 pacientes, $131(77,0 \%)$ foram diagnosticadas no estado pós-menopáusico. A Tabela 1 apresenta o risco de óbito associado ao grupo etário e nível de escolaridade medido por meio dos HR. Observa-se que não existiu diferença em relação à idade, porém houve tendência à diminuição do risco com o aumento do nível de escolaridade ( $\mathrm{p}$ para $\chi^{2}$ de tendência linear $=0,05$ ). 
Quanto ao tipo histológico, 157 (91,8\%) tumores eram do tipo carcinoma ductal infiltrante, outros sete $(4,1 \%)$ eram lobulares e seis se dividiram entre os tipos medular, mucinoso e metaplásico. A Tabela 2 apresenta a descrição das freqüências das variáveis relativas a características tumorais analisadas.

A função de sobrevida, no período de cinco anos, foi de $75,0 \%$, variando de $64,0 \%$ entre as que apresentaram metástase para linfonodo e de $84,0 \%$ para as que não apresentaram (Figura 1). Estratificando-se as pacientes segundo idade, observa-se que, embora as mulheres até 40 anos tenham aparentemente um resultado pior, se comparadas às com mais de 40 anos, a diferença entre as curvas não foi estatisticamente significante (Figura 2). Não foram constatadas diferenças em relação ao estado menopáusico e nutricional (dados não apresentados).

Ainda em relação às funções de sobrevida não ajustadas, observou-se que, com respeito às variáveis selecionadas relativas a características tumorais, as pacientes com tumores mais agressivos (pouco diferenciados) tiveram uma sobrevida bem inferior às com tumores menos agressivos (bem e moderadamente diferenciados) (Teste Log-rank, p = 0,003). Foi possível se observar ainda que as mulheres com receptores tumorais positivos para estrogênio tiveram uma melhor sobrevida (Teste Log-rank, $\mathrm{p}=0,025$ ). Não foram observadas diferenças estatisticamente significantes para o tamanho do tumor e para a presença de receptores tumorais positivos para progesterona.

A Tabela 3 apresenta os resultados do modelo de riscos proporcionais de Cox. Verifica-se que as HR brutas apontaram para um maior risco associado a pacientes com linfonodos comprometidos e com tumores mais agressivos. As pacientes com receptores tumorais positivos para estrogênio tiveram uma clara diminuição de risco. Na análise multivariada, na qual foram ajustadas no mesmo modelo todas as variáveis selecionadas e mais grupo etário e nível de escolaridade, o grau de agressividade do tumor deixou de ser estatisticamente significativo, e o risco de ter linfonodo comprometido ficou mais evidente ( $\mathrm{HR}=4,22$, IC95\%: 1,3712,98), assim como a diminuição de risco para aquelas que apresentaram receptores positivos para estrogênio foi mais expressiva $(\mathrm{HR}=0,12$, IC95\%: 0,02-0,73).

As variáveis analisadas não violaram o princípio de proporcionalidade de riscos; o resultado global do teste de resíduos de Schoenfeld apresentou $\mathrm{p}=0,39$ e não foi estatisticamente significativo para nenhuma das variáveis incluídas no modelo de riscos proporcionais de Cox.
Tabela 1

Risco de óbito associado à idade e ao nível de escolaridade.

\begin{tabular}{llll}
\hline Variável & $\mathbf{n}$ & HR & IC95\% \\
\hline $\begin{array}{l}\text { Faixa etária (em anos) } \\
30-39\end{array}$ & 14 & 1,00 & \\
$40-49$ & 41 & 0,32 & $(0,11-1,02)$ \\
$50-59$ & 44 & 0,40 & $(0,14-1,16)$ \\
$60-69$ & 45 & 0,36 & $(0,12-1,08)$ \\
70 e mais & 28 & 0,35 & $(0,10-1,19)$ \\
$\chi^{2}$ de tendência linear & & & $p=0,24$ \\
& & & \\
Nível de escolaridade & 21 & 1,00 & \\
Analfabeto & 71 & 0,78 & $(0,32-1,89)$ \\
Ensino elementar & 38 & 0,35 & $(0,11-1,14)$ \\
Ensino fundamental & 26 & 0,53 & $(0,16-1,68)$ \\
Ensino médio & 14 & 0,18 & $(0,02-1,49)$ \\
Ensino superior & & & $p=0,03$ \\
$\chi^{2}$ de tendência linear & & &
\end{tabular}

Tabela 2

Distribuição das pacientes segundo

características tumorais.

\begin{tabular}{|c|c|c|}
\hline Variável & $\mathbf{n}$ & $\%$ \\
\hline \multicolumn{3}{|c|}{ Tamanho do tumor $(\mathrm{em} \mathrm{cm})$} \\
\hline Até 1,0 & 7 & 4,12 \\
\hline $1,1-2,0$ & 47 & 27,65 \\
\hline $2,1-5,0$ & 76 & 44,71 \\
\hline 5,1 ou mais & 25 & 14,71 \\
\hline Sem informação & 15 & 8,82 \\
\hline \multicolumn{3}{|c|}{ Linfonodo comprometido } \\
\hline Não & 84 & 49,41 \\
\hline Sim & 86 & 50,59 \\
\hline \multicolumn{3}{|l|}{ Grau de Elston } \\
\hline I & 25 & 14,71 \\
\hline II & 89 & 52,35 \\
\hline III & 29 & 17,06 \\
\hline Sem informação & 27 & 15,88 \\
\hline \multicolumn{3}{|l|}{ Estrogênio } \\
\hline Negativo & 52 & 30,59 \\
\hline Positivo & 78 & 45,88 \\
\hline Sem informação & 40 & 23,53 \\
\hline \multicolumn{3}{|l|}{ Progesterona } \\
\hline Negativo & 60 & 35,29 \\
\hline Positivo & 70 & 41,18 \\
\hline Sem informação & 40 & 23,53 \\
\hline
\end{tabular}




\section{Figura 1}

Sobrevida segundo presença de linfonodo comprometido.

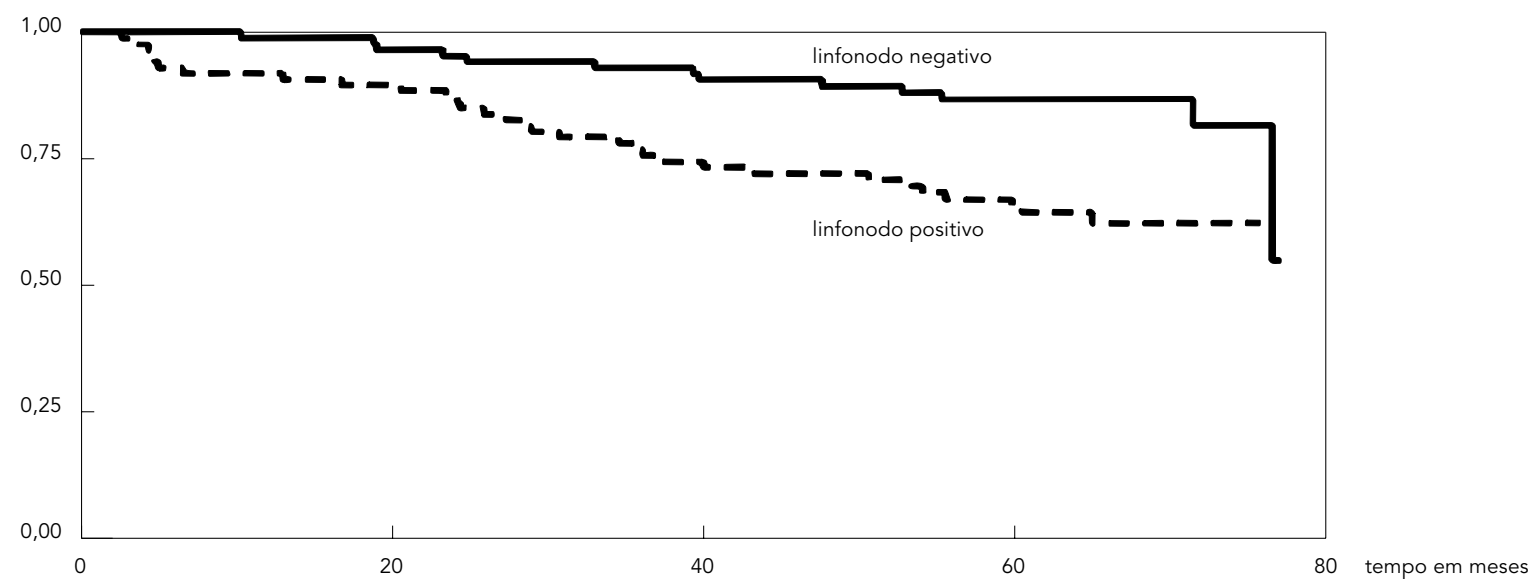

Teste Log-rank, $p=0,0009$

\section{Figura 2}

Sobrevida segundo grupo etário.

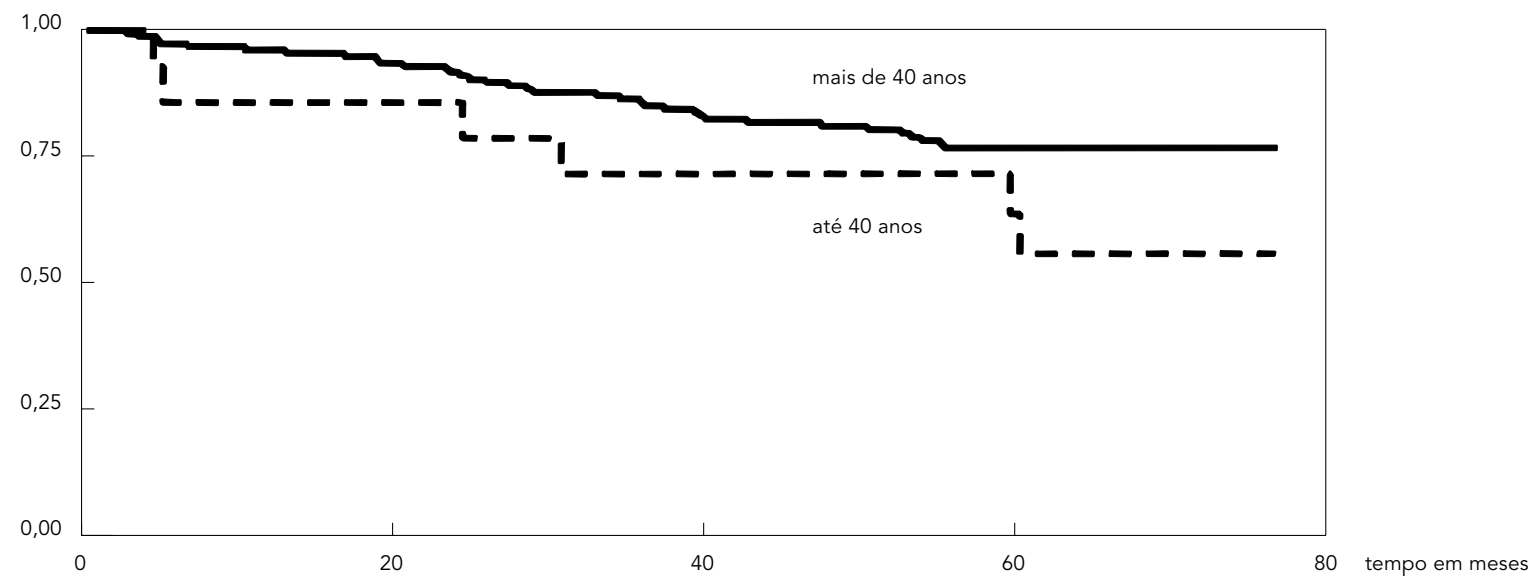

Teste Log-rank, $p=0,085$ 
Hazard ratios associadas a características tumorais.

\begin{tabular}{lcccc}
\hline Variável & HR bruta & IC95\% & HR $^{\star}$ & IC95\% \\
\hline Linfonodo comprometido & 3,04 & $1,53-6,08$ & 4,22 & $1,37-12,98$ \\
Tamanho do tumor $(>2 \times \leq 2 \mathrm{~cm})$ & 1,33 & $0,63-2,80$ & 0,91 & $0,32-2,56$ \\
Grau de Elston (III $\times$ I e II) & 2,65 & $1,35-5,19$ & 0,99 & $0,30-3,28$ \\
Receptor de estrogênio positivo & 0,42 & $0,19-0,92$ & 0,12 & $0,02-0,73$ \\
Receptor de progesterona positivo & 0,67 & $0,31-1,45$ & 1,32 & $0,30-5,86$ \\
\hline
\end{tabular}

* Ajustado por idade, nível de escolaridade e todas as variáveis da Tabela.

\section{Discussão e conclusão}

A sobrevida geral de cinco anos dessa coorte de mulheres estudadas foi de $75,0 \%$. Esse resultado é inferior ao encontrado por Chie et al. 16 na Tailândia (81,0\% para o mesmo período de tempo para casos incidentes recrutados entre 1991 e 1995). Estudo italiano que contou com 1.278 casos mostrou uma sobrevida de $86,9 \%$ para o estádio I e 63,3 para o estádio II 17. Pereira et al. 18 , analisando os dados de pacientes em coorte hospitalar em Belém, estimaram uma sobrevida em cinco anos de 61,0\%. Esse resultado inferior não pode, contudo, ser comparado diretamente com o presente estudo, pois, no primeiro, foram incluídos dados de todos os casos com diversas fases de estadiamento e não apenas para os casos cirúrgicos.

A existência de tendência linear à diminuição para as HR, na medida em que aumentava o nível de escolaridade, é concordante com o que vem sendo observado em outros estudos que mostram que o prognóstico das mulheres com câncer de mama tem relação inversa com o nível sócio-econômico 19,20. Possivelmente, mais do que os diferenciais absolutos no nível sócio-econômico, a influência do acesso aos serviços de saúde na prevenção e terapêutica poderia explicar essa relação inversa 21 . Metaanálise recente, baseada em dados de 14 estudos de sobrevida de câncer de mama, concluiu que mulheres afro-americanas comparadas a brancas têm um risco significativamente maior de morrer 22.

Chama a atenção o fato de que, na análise multivariada, na qual foram incluídos os fatores prognósticos relativos a características tumorais ajustados por idade e nível de escolaridade, apenas o grau do comprometido ganglionar se manteve como fator fortemente indicativo de pior prognóstico, e a presença de receptores positivos para estrogênio se associou à diminuição de risco de óbito.
Com certeza, ter linfonodo comprometido por metástase ainda é o principal preditor da evolução da doença, independentemente do tamanho do tumor. Chie et al. 16 apresentaram dados que confirmam essa afirmação; a sobrevida de pacientes com nódulo negativo foi muito maior quando comparadas às que tinham linfonodo negativo ( $\mathrm{HR}=3,65$, IC95\%: 2,33$5,71)$, independente do tamanho do tumor. O estudo realizado em Belém 18 mostrou uma sobrevida de $80,0 \%$ para mulheres sem metástase ganglionar, resultado bem superior ao índice global (61,0\%).

A associação entre grau de agressividade tumoral, definida segundo classificação de Elston, e risco de morte, comparando-se tumores pouco diferenciados (grau III) com os de grau I e II (bem e levemente diferenciados), quando controlada pela presença de metástase ganglionar, tamanho do tumor e receptores de estrogênio e progesterona, idade e nível de escolaridade, deixou de ser estatisticamente significante. Estudo realizado com parte das mulheres pertencentes a essa coorte 23 evidenciou que a presença de mutação no gene p53 em tecidos frescos de tumores era fortemente associada ao grau de Elston (OR $=11,97$, IC95\%: 2,24-307,05), esse resultado teve, porém, intervalo de confiança pouco preciso devido ao número pequeno de mulheres estudadas - apenas 51 mulheres. Estudo que analisou dados de 306 pacientes do INCA com carcinomas ductais infiltrantes de mama 24 , não evidenciou relação entre marcadores moleculares (c-erb B2, catepsina D, MIB-1, PCNA e p53) em cortes de parafina e existência de metástase para gânglios axilares. Esses achados, que a princípio podem parecer contraditórios, reforçam a idéia de que a participação de vários marcadores moleculares no prognóstico do câncer de mama ainda permanece inconclusiva, e a implementação de estudos que integrem dados epidemiológicos aos de biologia molecular e ge- 
nética é fundamental para o entendimento do processo de evolução dos tumores já iniciados.

Em relação aos receptores de estrogênio e progesterona, verificou-se que as mulheres que apresentavam receptor positivo para estrogênio tiveram melhor prognóstico, o mesmo não se evidenciou em relação aos receptores de progesterona. Embora muito estudado nos últimos vinte anos, o mecanismo pelo qual os receptores hormonais atuariam na evolução da doença ainda não é totalmente esclarecido. $\mathrm{O}$ valor prognóstico desses receptores varia de acordo com a idade, comprometimento ganglionar e tempo de seguimento após a cirurgia 25,26 . Estudo recente, que analisou a sobrevida de pacientes por mais de dez anos, mostrou que o benefício significativo dos receptores de estrogênio e progesterona aparece até cinco anos de seguimento; após esse período, as pacientes que apresentaram receptores positivos ou negativos tiveram quase o mesmo risco de sobreviver 27 .

\section{Resumo}

Vários fatores vêm sendo estudados com respeito ao estabelecimento de critérios mais seguros que influenciam o prognóstico de pacientes com câncer de mama. Este estudo teve como objetivo avaliar as taxas de sobrevida de cinco anos e os principais fatores prognósticos relativos ao tumor em mulheres com carcinoma invasivo de mama submetidas à cirurgia no Instituto Nacional de Câncer, Rio de Janeiro, Brasil, entre maio de 1995 e julho de 1996. As variáveis estudadas foram: presença de linfonodo comprometido, tamanho do tumor, grau de agressividade e presença de receptores hormonais para estrogênio e progesterona. As funções de sobrevida foram calculadas por meio do método de Kaplan-Meier. Foi utilizado o modelo de riscos proporcionais de Cox para avaliação dos fatores prognósticos. A taxa de sobrevida em cinco anos foi de 75,0\% para todas as pacientes e, de 64,0\% para as com metástase para linfonodo. A análise multivariada identificou o comprometimento de linfonodo como o mais forte preditor do desfecho; ter receptor positivo para estrogênio se associou a um melhor prognóstico. Esses resultados mostram a necessidade de condução de estudos que investiguem novos fatores que, combinados aos já conhecidos, possam melhor orientar a conduta terapêutica.

Neoplasias Mamárias; Prognóstico; Saúde da Mulher
Com base nos resultados encontrados, é possível se afirmar que, nessa coorte de pacientes estudas, os fatores preditores mais importantes para um melhor prognóstico seriam a ausência de metástase ganglionar e a presença de receptores de estrogênio. Isso reforça a posição de que é fundamental que os casos sejam diagnosticados e tratados ainda em estágios iniciais, o que aumentaria a chance da doença ainda não ter invadido linfonodos axilares. Por outro lado, a disponibilidade do teste de receptores hormonais tumorais deveria ser garantida para todas as pacientes, pois a nãoresposta poderia orientar, de forma mais precisa, a indicação de terapêuticas mais agressivas. Faz-se necessário, contudo, a implementação de estudos que possam investigar a presença de outros marcadores tumorais que, junto a outros dados epidemiológicos e clínicos, possam oferecer mais evidências para o estabelecimento de condutas terapêuticas eficazes para o câncer de mama.

\section{Colaboradores}

G. A. S. Mendonça concebeu e planejou o estudo, de construiu os bancos de dados, definiu as variáveis, analisou e interpretou os dados e redigiu o artigo. A. M. Silva participou da organização e manutenção dos bancos de dados, da análise estatística e da elaboração de tabelas e gráficos. W. M. Caula participou da coleta de dados em prontuários médicos e arquivo de Anatomia Patológica, da busca ativa por telefone para informação sobre pacientes e da organização das referências bibliográficas.

\section{Agradecimentos}

A toda a equipe médica e de enfermagem do Serviço de Mastologia do INCA pela cooperação durante a realização do estudo. Aos chefes dos Serviços de Arquivo Médico do INCA Ildalio Espinheira (INCA I) e Penha Cristina Motta Melo (INCA III) pelo empenho que tiveram na procura e disponibilidade dos prontuários médicos. Aos médicos patologistas Ana Lucia Eisenberg, Lídia Maria Magalhães Rezende, Paulo Antônio Faria e Sergio Romano pela ajuda na revisão e interpretação dos laudos histopatológicos. À Ângela Maria Cascão, chefe do Departamento de Dados Vitais da Secretaria de Estado de Saúde do Rio de Janeiro e sua equipe pela grande contribuição dada na busca de informação sobre óbito dos casos estudados. Este estudo foi financiado pelo CNPq (Bolsa de produtividade de pesquisa) e pela Universidade do Estado do Rio de Janeiro por meio de bolsas do Programa de Iniciação Científica. 


\section{Referências}

1. Parkin DM, Bray FI, Devesa SS. Cancer burden in the year 2000. The global picture. Eur J Cancer 2001; 37:S4-S66.

2. Ministério da Saúde. Atlas de mortalidade por câncer no Brasil 1979-1999. Rio de Janeiro: Instituto Nacional de Câncer; 2002.

3. Chu KC, Tarone RE, Kessler LG, Ries LA, Hankey BF, Miller BA, et al. Recent trends in U.S. breast cancer incidence, survival, and mortality rates. J Natl Cancer Inst 1996; 88:1571-9.

4. Quinn MJ, Martinez-Garcia C, Berrino F, The Eurocare Working Group. Variations in survival from breast cancer in Europe by age and country, 19781989. Eur J Cancer 1998; 34:2204-11.

5. Hunter CP, Redmond CK, Chen VW. Breast cancer: factors associated with stage at diagnosis in black and white women. J Natl Cancer Inst 1993; 85:1129-37.

6. Braud AC, Asselain B, Scholl S, Rochefordiere A, Palangie T, Dieras V, et al. Neoadjuvant chemotherapy in young breast cancer patients: correlation between response and relapse? Eur J Cancer 1999; 35:392-7.

7. Hortobagyi GN. Treatment of breast cancer. N Engl J Med 1998; 339:974-84.

8. Giuliano AE, Jones RC, Brennan M, Statman R. Sentinel lymphadenectomy in breast cancer. J Clin Oncol 1997; 15:2345-50.

9. Krag D, Weaver D, Ashikaga T, Moffat F, Klimberg VS, Shriver C, et al. The sentinel node in breast cancer: a multicenter validation study. N Engl J Med 1998; 339:941-6.

10. Boyages J, Delaney G, Taylor R. Predictors of local recurrence after treatment of ductal carcinoma in situ: a meta-analysis. Cancer 1999; 85:616-28.

11. Mendonça GAS, Eluf Neto J, Andrada-Serpa MJ, Carmo PAO, Barreto HCH, Inomata ONK, et al. Organochlorines and breast cancer: a case-control study in Brazil. Int J Cancer 1999; 83:596-600.

12. Elston CW, Ellis IO. Pathological prognostic factors in breast cancer. I. The value of histological grade in breast cancer: experience from a large study with long-term follow-up. Histopathology 1991; 19:403-10.

13. Armitage P, Berry G. Statistical methods in medical research. 2nd Ed. Oxford: Oxford Scientific Publications; 1987.

14. Kleinbaum DG. Survival analysis - a self-learning text. New York: Springer Verlag; 1997.

15. Cleves MA, Gould WW, Gutierrez RG. An introduction to survival analysis using Stata. College Station: Stata Press; 2002.

16. Chie WC, Chang SH, Huang CS, Tzeng SJ, Chen JH, Fan BY, et al. Prognostic factors for the survival of Taiwanese breast cancer patients. J Formos Med Assoc 2002; 10:98-103.
17. Russo A, Herd-Smith A, Gestri D, Bianch S, Vezzosi V, Del Turco MR, et al. Does family history influence survival of breast cancer patients? Int J Cancer 2002; 99:427-30.

18. Pereira WMM, Koifman S, Koifman RJ, Madeira A. Sobrevida de câncer de mama em coorte hospitalar em Belém, PA, 1993-95. Rev Bras Epidemiol 2002; Suplemento Especial:125.

19. Schrijvers CTM, Mackenbanch JP, Lutaz JM, Quinn MJ, Coleman MP. Deprivation, stage at diagnosis and cancer survival. Int J Cancer 1995; 63:324-9.

20. Polednak A. Survival of breast cancer patients in Connecticut in relation to socioeconomic and health care access indicators. J Urban Health 2002; 79:211-8.

21. Gorey KM, Holowaty EJ, Fehrubger G, Laukkanen E, Richter NL, Meyer CM. An international comparison of cancer survival: relatively poor areas of Toronto, Ontario and three US metropolitan areas. J Publ Health Med 2000; 22:343-8.

22. Newman LA, Mason J, Cote D, Vin Y, Carolin K, Bouwman D, et al. Afican-American ethnicity, socioeconomic status, and breast cancer survival: a meta-analysis of 14 studies involving over 10,000 African-American and 40,000 White American patients with carcinoma of the breast. Cancer 2002; 94:2844-54.

23. Simão TA, Ribeiro FS, Amorim LMF, Albano RM, Andrada-Serpa MJ, Cardoso LEB, et al. TP53 mutations in breast cancer tumors of patients from Rio de Janeiro, Brazil: association with risk factors and tumor characteristics. Int J Cancer 2002; 101:69-73.

24. Eisenberg ALA, Koifman S, Rezende LMC. Fatores preditivos para o envolvimento de linfonodos no câncer de mama. Rev Bras Cancerol 2001; 44:38996.

25. Collett K, Hartveit F, Skjaerven R, Maehle BO. Prognostic role of oestrogen and progesterone receptors in patients with breast cancer: relation to age and lymph node status. J Clin Pathol 1996; 49:920-5.

26. Hilsenbeck SG, Ravdin PM, De Moor CA, Chamness GC, Osborne CK, Clark GM. Time-dependence of hazard ratios for prognostic factors in primary breast cancer. Breast Cancer Res Treat 1998; 523:227-37.

27. Costa S-D, Lange S, Klinga K, Merkle E, Kaufmann $M$. Factors influencing the prognostic role of oestrogen and progesterone receptor levels in breast cancer - results or the analysis of 670 patients with 11 years of follow up. Eur J Cancer 2002; 38:1329-34

Recebido em 16/Jan/2003

Versão final reapresentada em 08/Jan/2004 Aprovado em 09/Mar/2004 